\title{
Syntaxe et valeur discursive de la construction et VS en anglo-normand par rapport au français du continent.
}

\author{
Ingham, Richard \\ Birmingham City University \\ richard.ingham@bcu.ac.uk
}

\section{Introduction}

Dans cette intervention nous abordons la question de la transmission du français en Angleterre vers 1300. La pratique de la langue française, on le sait, a été introduite dans l'île par la conquête normande du XIème siècle ; elle a nourri une littérature florissante pendant deux siècle à peu près (Vising 1923, Legge 1950, Dean 1993). A partir de la seconde moitié du $13^{\text {ème }}$, le français est devenu une langue quasi-officielle utilisée à des fins administratives à coté du latin. Mais sa longue survie, surtout à une époque où, hors la cour royale, pratiquement toute la population était de langue maternelle anglaise, ${ }^{1}$ pose le problème de savoir comment il s'est reproduit d'une génération à l'autre pendant au moins trois siècles, et surtout lorsqu'il ne bénéficiait plus, au bout de trois à quatre générations, du statut de langue maternelle de la classe gouvernante. Par le système scolaire, selon l'opinion de certains, comme Rothwell (1976). Cependant, les écoles anglaises, comme partout dans l'Europe du moyen âge, n'avaient comme vocation que l'enseignement du latin (Leach 1915, Orme 1973), donc cette forme d'explication semblerait peu prometteuse. ${ }^{2}$

Selon Kibbee (1996) il y aurait eu une différence capitale entre le français du continent et celui de l'Angleterre, qui abondait en emplois fautifs et en constructions calquées sur celles de l'anglais. Le français insulaire serait devenu, au bout d'un siècle ou deux, une variété artificielle, coupée de ses bases et ne participant plus aux grands courants de l'évolution du français. C'est une notion qui a été vivement rejetée par Trotter (2003), qui montre dans ses recherches sur l'emploi quotidien du français en Angleterre médiévale qu'il continuait à servir de moyen de communication tout le long du XIVème siècle ; tant à l'intérieur qu'à l'extérieur du pays. En effet, si le français insulaire était devenu un «jargon à peine compris par ses usagers ", selon l'expression de Pope (1934), on ne comprend pas en effet pourquoi les Anglais auraient voulu continuer à s'en servir pour communiquer entre eux. Pour Trotter, le français insulaire faisait partie de l'aire dialectale du français médiéval, ne serait-ce qu'en raison des réseaux culturels et commerciaux qui reliaient les deux pays, nécessitant des moyens de communication tant soit peu adéquats.

Citons à ce propos des travaux réalisés par Ingham $(2006 \mathrm{a}, 2006 \mathrm{~b}, 2011)$ sur la syntaxe de l'anglo-normand tardif, et indiquant que cette variété a participé aux innovations syntaxiques du français survenues vers 1300 environ. Le français continental a adopté un système mixte de verbe second dans les principales : la postposition du sujet suivant un complément d'objet direct antéposé demeurait la règle, alors que l'ordre XSV s'employait librement suivant un complément circonstanciel, et surtout temporel (Vance 1997). Aux XIIIème-XIVème siècles, l'indefini aucun s'est introduit au profit de $n u l$, d'abord dans les énoncés virtuels, et ensuite dans les négatives, en anglo-normand tout comme en français de France (Ingham 2011). En outre, la syntaxe d'un pronom complément d'objet direct régi par un verbe à l'infinitif s'est modifiée vers la même époque, de sorte que désormais le pronom atone devait précéder le verbe à l'infinitif au lieu de le suivre, comme cela avait été le cas en ancien français (de Kok 1985).

La précision frappante avec laquelle le français insulaire a suivi l'évolution du français dans ces domaines tend à appuyer la thèse de Trotter, selon qui l'anglo-normand appartenait encore à cette époque au continuum dialectal francophone. Signalons que les traits grammaticaux examinés par Ingham ont été sélectionnés afin de bien cerner le type de règle grammaticale qui est censée poser un défi particulier aux apprenants d'une langue étrangère, c'est-à-dire que dans ces cas, la syntaxe de la langue cible et celle de la langue maternelle divergent. Les usagers de l'anglo-normand ont néanmoins réussi à reproduire les changements syntaxiques survenus dans le français du continent, sans se laisser influencer par la grammaire anglaise. Il semblerait 
d'après ces résultats que la notion selon laquelle l'anglo-normand, soumis à l'influence de l'anglais, se serait dissocié du français du continent est sujette à caution, du moins en ce qui concerne sa syntaxe.

Dans cette étude nous développons ce thème du parallélisme syntaxique entre le français insulaire et celui de France, à propos d'une règle syntaxique qui met en jeu non seulement l'ordre des mots, mais également un corrélat pragmatique, c'est-à-dire que les locuteurs anglophones devaient appréhender les conditions discursives dans lesquelles on employait la construction en question. Comme on verra, adopter cette optique pourra nous renseigner plus amplement sur le contexte où les anglais apprenaient le français à cette époque.

\section{L'ordre et VS en ancien français}

L'ordre et-Verbe fléchi-Sujet nominal (et VS), illustré par les exemples en (1) et (2), constitue un trait caractéristique du moyen français des XVe et XVIe siècles Bergh (1952), Baulier (1956), Lemieux (1991), et Vance (1993): ${ }^{3}$

(1) Et seront le pere et le filz condamnés a etre penduz

Jean d'Arras, Melusine 226, 23

(2) Et conduisit Melusine la contesse et sa fille jusques oultre la villete de Coulombiers Jean d'Arras, Mélusine 206, 26

Ce qui est moins bien établi est la chronologie du phénomène, et la question est de savoir si elle possédait dès ses débuts la valeur discursive particulière qu'elle avait par la suite. Prévost (2001) a fait valoir l'effet pragmatique de la postposition du sujet en moyen français des années 1450-1550. Combettes (1988) indique également qu'à cette époque, où le caractère TopiqueVerbe-Sujet de l'ancien français commençait à s'estomper, la dimension discursive reste importante quant à la postposition du sujet. Pour sa part, Bergh (1952), qui a situé la montée de et VS aux environs de 1350, a proposé que et VS en moyen français représentait une tournure stylistique qui servait à «animer le récit» et à «donner plus d'entrain aux différentes phases de l'action». Ce lien entre le sémantisme discursif de et et la postposition du sujet a été repris et développé par Prévost (2001) qui, dans une étude de textes en prose écrits entre 1450-1550, a observé dans l'emploi de et VS un effet de «surenchère informative» :

(3) «Et [à sujet postposé] traduit toujours une forme de continuité thématique [...] mais il introduit en même temps un énoncé qui a un caractère inattendu dans la mesure où il constitue une surenchère informative. Or on ne relève pas d'opération similaire lorsque le sujet est préverbal.» (Prévost 2001, 279)

Son approche n'est pas sans rappeler l'affirmation de Buridant $(2000,553)$ selon qui en ancien français «et peut marquer une addition insistante au sens de 'et de surcroît' ». Il reste qu'aucun de ces auteurs n'a poursuivi la question des origines de la construction. Vance (1993) a signalé la présence déjà en ancien français de l'ordre VS suivant et, bien que l'étendue du phénomène paraisse avoir été encore assez restreinte. Ses travaux ne permettent cependant pas de savoir si les tendances discursives observées par Prévost dans les textes en moyen français se manifestaient dès l'époque qui l'a précédé.

Dans cet exposé nous résumons d'abord l'analyse d'Ingham (2007) que nous allons renforcer à l'aide de données supplémentaires tirées de textes continentaux, avant d'aborder le problème de l'anglo-normand: dans quelle mesure a-t-il suivi l'évolution du français quant à l'emploi de et VS ?

Seront distingués deux types d'énoncés, dont le premier, désigné par le terme «inaccusatif» (Legendre 1989) comprend soit un groupe verbal à la voix passive, p. ex. (1), soit un verbe ayant un caractère sémantique de déplacement ou de transformation (Junker 1990), tels aler, venir, arriver, partir, mourir, muer, ou bien une signification aspectuelle (commencer, durer, cesser). Selon cette perspective théorique, le sujet superficiel du verbe possède le statut d'un objet sous-jacent (v. Legendre 1989 et références cités). Le second groupe sera constitué des 
autres énoncés, où figurent surtout des verbes agentifs et autres verbes transitifs, p. ex. (2), que l'on désigne par le terme 'inergatif'.

A été exclu du compte tout énoncé où figure le connecteur et comportant soit (i) un sujet pronominal (personnel, démonstratif, quantificateur ou indéfini, p. ex.: il, cil, uns, chacun), soit (ii) deux verbes coordonnés, où la postposition du sujet aurait compliqué la construction, soit (iii) un verbe pronominal, puisque dans ce cas le sujet grammatical peut être à la fois agent et patient de l'action, ce qui brouillerait la distinction voulue entre verbes inaccusatifs et autres.

\section{Textes sources}

Pour faire une étude de corpus de l'évolution de la syntaxe de l'ancien et du moyen français, on dispose actuellement de ressources plutôt abondantes par rapport à la situation d'il y a quelques années. Les nombreux textes littéraires réunis par la BFM et par le NCA recouvrent la quasitotalité de l'époque ciblée. Cependant, pour réaliser une étude syntaxique il vaut mieux se servir de textes en prose, pour éviter que les effets de la rime et de la métrique ne perturbent les données obtenues, et les textes en prose sont peu nombreux dans les bases de corpus, avant l'ère du moyen français. ${ }^{4}$ La disparité de genres constitue un autre facteur susceptible de fausser les résultats d'une étude quantitative, dans la mesure où certains genres sont plus conservateurs que d'autres, et que certains genres sont mieux représentés à certaines époques qu'à d'autres.

En dehors des corpus, cependant, à l'époque qui nous intéresse, qui va de 1230 à 1350 environ, on dispose d'un bon nombre de chroniques en prose, qui vont nous permettre d'établir une série de textes appartenant plus ou moins au même genre textuel. Ceux-ci présentent en outre l'avantage d'exister dans des éditions réalisées à partir de manuscrits qui ne sont pas postérieurs de beaucoup à la date de composition de l'œuvre concernée, détail important en ce qui concerne la capacité d'un texte à témoigner de la conjoncture linguistique de telle ou telle époque. Il s'agit d'abord de la Chronique d'Ernoul, qui remonte aux environs de 1230, éditée par de Mas Latrie d'après un manuscrit du XIIIe siècle, et des Grandes Chroniques des Rois de France (GCRF), dont la composition de la première partie, attribuée à un religieux qui de toute apparence s'appelait Primat, se situe aux environs de 1270-1275. Cette chronique a été publiée par Viard sur la base d'un manuscrit remontant à la fin du XIIIème. Pour le présent travail, nous avons effectué un dépouillement des données fournies par les tomes I, II et VI; pour ce qui est des successeurs de Primat, on s'est servi des tomes VII (la partie consacrée à la vie de S. Louis, qui figure dans une source manuscrite du début du XIVème siècle) et IX (la chronique de Philippe VI de Valois, rédigée peu après la mort de celui-ci en 1350, et éditée à partir d'un manuscrit de 1380 environ).

La série de chroniques est prolongée dans la première moitié du XIVème siècle par les continuations des GCF, de la Chronique Artésienne, et de la Chronique de Jean le Bel. Au total, nous avons pu réunir un ensemble de huit textes historiographiques témoignant de la langue de cette période charnière, qui enjambe les dernières décennies de l'ancien français et les débuts du moyen français.

\section{Analyse syntaxique de et VS, 1230-1340, chroniques françaises}

Notre analyse syntaxique portera à la fois sur les fréquences du connecteur et suivi soit de VS, soit de SV, en l'absence d'un élément thématique précédant le verbe, et aussi sur la forme de l'inversion avec et VS; il s'agit de savoir si ces textes annoncent déjà l'inversion dite " libre ", trait caractéristique du Moyen Français, par où le sujet grammatical est postposé à l'ensemble du groupe verbal (Prévost 2001: 131), p. ex. (4a), ou bien s'ils se limitent à l'inversion dite " germanique », par où le sujet grammatical s'intercale entre le verbe fléchi et le reste du groupe verbal, p. ex. (4b) :

(4)a. Et ceste chose a faicte ly roys.

(4)b. Einsint fu li ponz refez.
Mélusine p. 224

Villehardouin $§ 163$ 
A été exclu du compte tout énoncé où figure le connecteur et comportant soit (i) un sujet pronominal (personnel, démonstratif, quantifieur ou indéfini, p. ex.: il, cil, uns, chacun), soit (ii) deux verbes coordonnés, où la postposition du sujet aurait compliqué la construction, soit (iii) un verbe pronominal, puisque dans ce cas le sujet grammatical peut être à la fois agent et patient de l'action, ce qui nous empêcherait de tirer la distinction voulue entre verbes inaccusatifs et verbes inergatifs.

Les relevés de fréquence de et VS obtenus dans les textes examinés sont donnés dans le Tableau 1. Au cours des cent ans environ séparant la Chronique d'Ernoul de la GCRF IX, l'on observe une très forte montée de la postposition du sujet suivant le connecteur et. La postposition s'avère très productive dès la GCRF VII, composée aux alentours de 1300, et dans les contextes inaccusatifs elle est même majoritaire.

Selon le témoignage de ces textes, la percée de la postposition du sujet après et se fait largement sentir dans la deuxième moitié du XIIIème. Avant cette date, la construction restait rare avec les verbes inaccusatifs et presqu'inexistante avec les verbes inergatifs.

Tableau 1 : Occurrences de et VS, et et $\mathrm{SV}$ dans les propositions principales à sujet nominal débutant par et, français du continent

\begin{tabular}{|c|c|c|c|c|c|c|}
\hline & \multicolumn{3}{|c|}{ verbes inaccusatifs } & \multicolumn{3}{|c|}{ verbes inergatifs } \\
\hline & $\mathrm{VS}$ & SV & total & VS & $\mathrm{SV}$ & total \\
\hline Chron. Ernoul & 4 & 45 & 49 & 0 & 70 & 70 \\
\hline Hist. Ducs Norm. & 5 & 23 & 28 & 0 & 29 & 29 \\
\hline GCF I-VI & 11 & 28 & 39 & 5 & 72 & 77 \\
\hline $\begin{array}{l}\text { Villehardouin Ms B } \\
\text { (pp. 42-240) }\end{array}$ & 35 & 71 & 106 & 6 & 68 & 74 \\
\hline GCRF VII & 28 & 8 & 36 & 13 & 19 & 32 \\
\hline Chronique Artésienne & 39 & 16 & 55 & 20 & 21 & 41 \\
\hline GCRF IX & 70 & 18 & 88 & 25 & 13 & 38 \\
\hline J. le Bel I ( $1^{\text {ère }}$ partie $)$ & 22 & 6 & 28 & 16 & 9 & 25 \\
\hline Total & 214 & 215 & 429 & 85 & 301 & 386 \\
\hline
\end{tabular}

Ces chiffres sont repris en termes de pourcentage dans la Figure 1 : 
Figure I : Pourcentages de et VS dans les propositions principales à sujet nominal débutant par et, français du continent

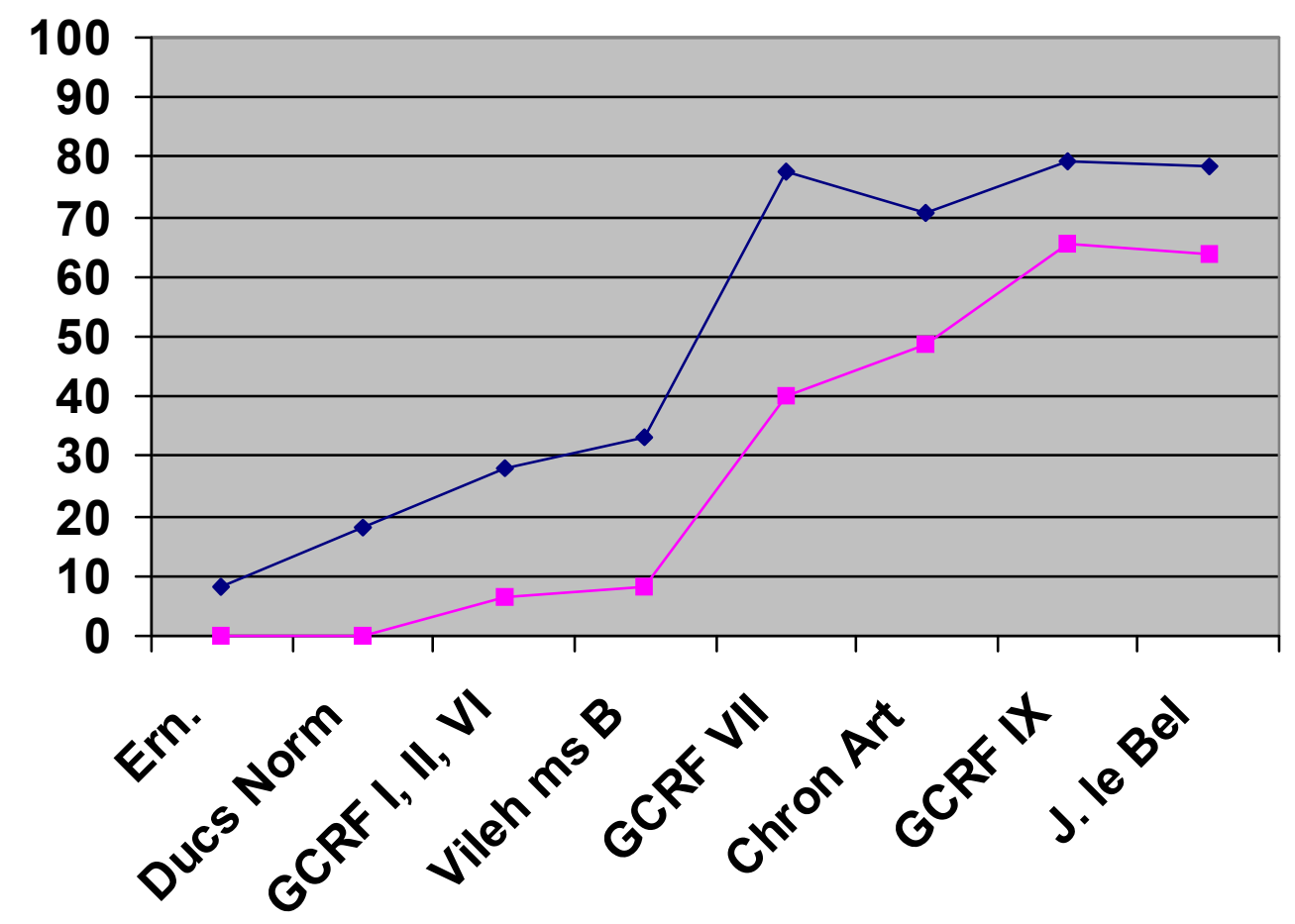

\section{$\rightarrow-$ VS inacc $\rightarrow$ VS inerg}

\section{La valeur discursive de et VS par rapport à et SV}

Examinons l'emploi de [et VS] dans les texte le plus tardifs, c'est-à-dire la GCRF IX et la chronique de Jean le Bel, qui sont les plus rapprochés de ceux étudiés par Prévost (2001). Dans ces deux ensembles de textes, les énoncés en et VS autres qu'inaccusatifs s'accompagnent très souvent de la notion d'une surenchère informative, p. ex.:

(5)a. ... I homme qui se disoit avoir este pris des anemis et puis ranconne, lequel disoit ...que le roy d'Angleterre faisoit faire moult diligeaument le pont de Poyssi, et vouloit celui homme recevoir mort s'il ne disoit verite.

GCRF IX 278, 3

(5)b. Ledit messier Robert fu navre et fu au lit, et le prist I flux de ventre duquel il mouroit assez tot. GCRF IX 228, 8

A chaque fois, l'interprétation «et de surcroît» est pragmatiquement naturelle. Lorsqu'il s'agit d'une précision qui n'apporte rien d'inattendu, par contre, on observe plutot et SV, p. ex.:

(6) Adoncques le conte de Moret [...fu pris des Anglois quant il retornoit.... Et ledit conte de Moret fu mene en une des prisons au roy d'Angleterre.

GCRF IX 151, 21

(7) ... ne voult pas monseigneur Robert d'Artois passer avec le roy [sc. Edouard III d'Angleterre] pour ce qu'il pensoit que le roy feroit correction quant il vendroit en Angleterre de eulz qui avoient ainsi les deniers detenuz, et ledit messier Robert d'Artois ne vouloit point avoir le maugré..

GCRF IX 210, 3

Dans les exemples (6) et (7), il s'agit bien, pour rappeler les termes de Prévost (2001, 277), d'un événement qui n'est ni «inattendu ni surprenant», et donc d'une «neutralité dans l'enchaînement». 
Il est à noter cependant qu'en présence d'un verbe inaccusatif, et surtout d'un verbe à la voix passive, la valeur discursive de surenchère de la tournure et VS est loin d'être garantie dans la GCRF IX. On y rencontre même un certain nombre de cas où la signification discursive permet au contraire l'interprétation «et bien sûr», par exemple en (8) :

(8) Et puis se departirent touz fors le duc de Breban, auquel l'en monstra que trop seroit son filz bas marie a la fille le conte de Haynau, et trop plus grant honneur seroit que il preist la fille au roy de France. Tantost le duc s'i acorda et fu depecie le mariage de la fille au conte de Haynau et du filz au duc de Brebant.

GCRF IX 127, 14

L'annulation du mariage prévu, exprimée dans l'énoncé et VS débutant par et fu depecie... n'apporte pas de surenchère informative, puisque l'accord du duc à cette proposition a déjà été évoqué dans la phrase précédente.

L'ordre et SV s'employait chez Jean le Bel lorsqu'il ne s'agissait pas de surenchère informative, mais plutôt du type d'information qui n'apporte rien d'inattendu, p. ex. :

(9) Ainsy disoient les Francoys que se vouloient attribuer l'honneur de cele departie, et les Anglois maintenoient le contraire par les raisons dessusdites.

Jean le Bel XXXII, 165

En somme, les textes continentaux de la mi-XIVème siècle font apparaître les mêmes tendances que celles constatées par Prévost (2001) pour le moyen français du XVème. Déjà, au début du moyen français, et donc à une époque où le français continuait à s'employer couramment en Angleterre, la tournure en question possédait sa valeur de surenchère informative. Ainsi, on pourra poser la question de savoir si elle s'est répandue en français insulaire comme sur le continent.

\section{La construction en et VS en anglo-normand}

Comme pour le français continental, nous avons réuni un ensemble de textes historiographiques. Compte tenu de l'évolution diachronique déjà notée dans les textes du continent (voir ci-dessus, figure no. 1), on les a repartis en deux périodes, correspondant aux XIIIème et XIVème siècles. Etant donné le nombre réduit de sources disponibles, par rapport à la profusion de chroniques continentales de l'époque, nous avons admis dans notre choix de textes anglo-normands deux oeuvres qui ne sont historiographiques qu'au sens large, c'est-à-dire la Chronique pseudoTurpin dans la version insulaire de Brian (deuxième moitié du XIIIème) et Fouke le Fitz Waryn, mélange de roman et de relation historique redigé entre 1320-1340, à peu près. Les autres textes sont des chroniques, dont les plus importantes sont Le livre des Reis d'Engeleterre, le Brut en prose, tous deux de la fin du XIIIème siecle environ, et l'Anonimalle Chronique et la Chronique de Londres, du milieu du XIVème.

La construction et VS se rencontre en effet dans ces textes insulaires, mais de façon très inégale: elle n'a existé qu'en présence d'un verbe inaccusatif, comme on peut le constater dans le tableau no. 2 :

Tableau 2 : Occurrences de et VS, et et SV dans les propositions principales à sujet nominal débutant par et, français insulaire

\begin{tabular}{|l|c|c|c|c|c|c|}
\hline & \multicolumn{2}{|c|}{ Verbes inaccusatifs. } & \multicolumn{2}{c|}{ Verbes inergatifs } \\
\hline & VS & SV & T & VS & SV & T \\
\hline Tripartita & 0 & 9 & 9 & 0 & 4 & 4 \\
\hline $\begin{array}{l}\text { Turpin anglo- } \\
\text { normand }\end{array}$ & 5 & 7 & 12 & 0 & 14 & 14 \\
\hline Feudal manual & 7 & 6 & 13 & 0 & 10 & 10 \\
\hline LRB & 1 & 2 & 3 & 0 & 6 & 6 \\
\hline LRE & 3 & 20 & 23 & 0 & 18 & 18 \\
\hline Brut (Marvin) & 0 & 19 & 19 & 0 & 36 & 36 \\
\hline $\begin{array}{l}\text { Total période I } \\
(c .1200-c .1300)\end{array}$ & 16 & 63 & 79 & 0 & 88 & 88 \\
& $(20.3 \%)$ & $(79.7 \%)$ & & & & \\
\hline
\end{tabular}




\begin{tabular}{|l|c|c|c|c|c|c|}
\hline & & & & & & \\
\hline Wrox./Semp. & 4 & 12 & 16 & 0 & 11 & 11 \\
\hline FFW & 1 & 24 & 25 & 0 & 25 & 25 \\
\hline AC-> 1333 & 8 & 45 & 53 & 0 & 32 & 32 \\
\hline CL & 7 & 19 & 26 & 0 & 31 & 31 \\
\hline AC 1334- 1356 & 4 & 10 & 14 & 0 & 15 & 15 \\
\hline $\begin{array}{l}\text { Total période II } \\
(c .1305-c .1375)\end{array}$ & 24 & 110 & 134 & 0 & 114 & 114 \\
$(18 \%)$ & $(82 \%)$ & & & $(100 \%)$ & \\
\hline
\end{tabular}

À la différence du français continental, il ne s'est manifesté en anglo-normand aucune tendance diachronique ni dans la fréquence ni dans la distribution de et VS. Avec les verbes inaccusatifs, la construction en et VS a plafonné autour d'un niveau de $20 \%$ pendant les deux périodes. Pour ce qui est des verbes inergatifs, et VS est resté entièrement absent dans les périodes étudiées, même lorsque le contexte est celui d'une surenchère informative, $p$. ex. :

(10) Quar pur la grant promesse le roy me volez vous gerpyr, e le roy vous ad maundee une letre laquele, sire, vous avez celee de moy.

FFW 39

(11) Bien tost vindrent sis gros e grantz vilanynz e chescun avoit en sa meyn un gros bastoun dur e fort.

FFW 43

(12) As queux noeces ycesti roi ove Isabelle sa compaigne et plusours graunz de cest terre feurent. Et cesti roi Edward despendist illuqes a ceo qe fu dist plus de lx mille livres. AC 2,86

Dans tous ces cas, on imagine facilement qu'un texte continental du XIVème siecle aurait bien pu employer et VS. À chaque fois, la proposition introduite par et fournit une précision importante que l'on n'aurait pas pu prévoir: la lettre cachée dont il est question dans l'exemple (10), le 'gros bâton' de l'exemple (11), et enfin l'énormité de la somme dépensée dans l'exemple (12).

De plus, les quelques occurrences de et VS avec un verbe inaccusatif que l'on observe ne respectent pas la contrainte discursive en question, comme dans les exemples suivant où le sujet est à chaque fois un référent entièrement nouveau ('une bourse', 'chanoines') :

(13) Et fut trovée un bourse, et en cele bourse une piece de sendal rouge, en la quele furent envolupez ossementz de les $\mathrm{xj}^{\mathrm{m}}$. virgines

CL 45

(14) E vindrunt iloec chanoignes de iii luys

LRE 238,14

Les occurrences de et VS se présentent le plus souvent avec les verbes être ou durer, p.ex. :

(15) Et dura la graunt chierte de bledz continulment par trois annz

AC 90,20

(16) Et fu lour_homage rendu a li par monsire William Trussell

AC 132,19

On continuait d'employer et SV ou et VS indifféremment, sans respecter les considérations de «neutralité dans l'enchaînement» ou de la «surenchère informatique» signalées par Prévost (2001).

Il reste à comprendre pourquoi l'anglo-normand s'est décroché de l'évolution de la francophonie médiévale précisément dans ce domaine-ci, alors qu'à bien d'autres égards, (Ingham 2006a,b, 2011), il a adopté les nouvelles tendances qui se sont imposées dans le passage de l'ancien au moyen français.

\section{Explication psycholinguistique}

Pour comprendre le manque d'évolution de la construction en et VS au XIVème siècle, il convient de prendre en compte l'aspect psycholinguistique de l'anglo-normand vers 1300 . Sa 
transmission se faisait le plus souvent par le biais de l'école, non comme matière scolaire, mais comme langue véhicule utilisée pour l'apprentissage du latin, que l'on abordait à l'âge de 7 ans. Pour cette raison, on a dû apprendre le français dès le début de la scolarité, c'est-à-dire à partir de 5 ans (Orme 1973). Il s'agissait sûrement d'un contexte d'immersion, de bain linguistique, qui permettait aux usagers du système scolaire anglais d'apprendre le français de manière orale, en l'absence de matériaux - livres de grammaire et de vocabulaire - voués à l'enseignement du français par l'écrit, qui n'ont fait leur apparition que vers la fin du XIVème siècle (Lusignan 1986).

Puisque à d'autres égards l'anglo-normand a bien suivi la direction du changement syntaxique en français, il faut se demander pourquoi les usagers du français insulaire du XIVème ne l'ont pas fait dans le cas de et VS. L'explication proposée est que cette tournure était sujette à un conditionnement discursif qui se laissait difficilement appréhender par les apprenants anglophones, dans les conditions qui prévalaient à l'école. Le propre de cette construction était de s'employer, on l'a vu, dans un contexte discursif bien déterminé, celui du récit. Or, à l'école le mode discursif dominant était celui de l'exégèse de textes, souvent par la traduction latinfrançais, ou vice versa, non pas celui de la narration, du moins en langue française. Pour cette raison, les occasions ont dû manquer au cours desquelles les jeunes apprenants du français auraient pu se familiariser avec de nouveaux courants dans l'emploi discursif de la syntaxe française. Les autres changements grammaticaux adoptés par l'anglo-normand signalés plus haut, par contre, s'employaient dans pratiquement tous les genres discursifs; le locuteur français, même dans le contexte assez limité du milieu scolaire, a donc pu s'en servir couramment, et ainsi fournir un modèle qu'ont pu imiter les jeunes apprenants, comme cela se passe d'ordinaire dans le cadre du bain linguistique.

\section{Conclusions}

La comparaison effectuée dans cette étude de la syntaxe des énoncés commençant par et aux débuts du moyen français nous a permis de dégager un trait important et jusqu'ici ignoré de l'anglo-normand tardif. Celui-ci, tout en adoptant de façon générale les innovations syntaxiques du français survenues vers 1300 , n'a pas suivi le mouvement lorsqu'il s'agissait d'une tendance qui mettait en cause non seulement la syntaxe distributionnelle, mais également la valeur pragmatique/discursive d'une construction. Les locuteurs anglo-normands ont continué d'employer la tournure en et VS uniquement avec des verbes inaccusatifs, sans adopter la nouvelle tendance du français de France à utiliser et VS avec des verbes inergatifs, sujet à une contrainte discursive bien déterminée. Ce décalage manifeste entre l'adoption de nouvelles formes syntaxiques d'un côté, et la résistance a l'innovation de l'autre, nous incite a considérer sous quels aspects le français insulaire a été transmis a cette époque, et à mettre en cause les modalités de son emploi en tant que langue véhicule dans le contexte du système scolaire anglais médiéval. On sait que la syntaxe du français du moyen âge montre très peu de variabilité syntaxique ; si l'on en trouve précisément dans le domaine d'une construction conditionnée par des facteurs discursifs, il semblerait bien que la survie d'une variété linguistique, comme celle du français exporté en Angleterre, et les formes que prend cette variété, doivent dépendre étroitement des conditions de sa transmission.

\section{Textes continentaux}

Chronique Artésienne, éd. F. Funck-Brentano. Paris : A. Picard, 1899.

La Chronique d'Ernoul et de Bernard le Trésorier, éd. L. de Mas Latrie. Paris, Champion 1871.

Chronique de Jean le Bel, éd. J.Viard et E. Déprez, 2 t. Paris, 1904.

Geoffroy de Villehardouin, La conquête de Constantinople, éd. J. Dufournet, Paris, Flammarion, 2004.

Les Grandes Chroniques de France, éd. J. Viard, t. I, II, VII et IX, Société de l'Histoire de France, Paris, Champion, 1920-1937.

Histoire des ducs de Normandie et des rois d'Angleterre, éd. F. Michel. Paris: Société de 1'Histoire de France, 1840.

Jean d'Arras, Mélusine ou La noble histoire de Lusignan, roman du XIVe siécle, éd. J.-J. Vincensini, Paris, Livre de Poche, 2003. 


\section{Textes anglo-normands}

'Pseudo-Turpin anglo-normand': The Anglo-Norman pseudo-Turpin Chronicle of William de Briane, éd. I. Short. ANTS 25, 1973.

'AC - 1333': The Anonimalle Chronicle 1307-1334, éds. W. Childs \& J. Taylor, Yorkshire Archaeological Society record series 147, 1991.

'AC 1334-56' The Anonimalle Chronicle 1333-1381, éd. V. Galbraith. Manchester University Press.

'Brut' : The earliest anglo-norman prose Brut, éd. J. Marvin, Cambridge, Boydell, 2006.

'CL': Chroniques de London depuis l'an 44 Hen. III jusqu'à l'an 17 Edw. III, éd. G. Aungier. Camden Society, 1844

'Feudal Manual': Feudal manuals of English history, éd. T. Wright .J. Mayer: Liverpool, 1872.

'FFW': Foulke le Fitzwarine éds. E. Hathaway, P. Ricketts, C. Robson, and A. Wiltshire, ANTS. Oxford: Basil Blackwell, 1975

'LRB, LRE: Le livere de reis de Brittanie e le livere de reis de Engleterre, éd. J. Glover, Rolls series no. 42. London: Longmans, 1865.

'PROME': 'Tripart': Tyson, D. An early French prose history of the Kings of England. Romania (1975) $96,1-26$.

\section{Références bibliographiques}

Baulier, F. (1956). Contribution à l'étude de l'inversion du sujet après la conjonction et. Le Français Moderne 24, 250-257.

Bergh, L. (1952). Quelques réflexions sur l'inversion après la conjonction et en Ancien et en Moyen Français. In Mélanges K. Michaëlsson, Göteborg, pp. 43-66.

Buridant, C. (2000). Grammaire nouvelle de l'ancien Français. Paris: Sedes.

Combettes, B.(1988). Recherches sur l'ordre des éléments de la phrase en moyen Français. Thèse pour le Doctorat d'Etat, Université de Nancy.

Dean, R. (1999). Anglo-Norman Literature: A Guide to Texts and Manuscripts. ANTS Occasional Publication Series 3. Londres: ANTS.

Guillot, C. (2010). CORPTEF: Corpus représentatif des premiers textes français. ENS Lyon.

Ingham, R. (2006a). Syntactic change in Anglo-Norman and Continental French Chronicles: was there a 'Middle' Anglo-Norman? Journal of French Language Studies 16/1, 25-49.

Ingham, R. (2006b). The status of French in medieval England: evidence from the use of object pronoun syntax. Vox Romanica 65, 1-22.

Ingham, R. (2007). Le changement linguistique et l'oralité du texte : la syntaxe du connecteur et aux débuts du moyen français. In A. Vanderheyden, J. Mortelmans, W. de Mulder et T. Venckeleer (éds) Texte et discours en moyen français. Tournai : Brepols.

Ingham, R. (2011). Grammar change in Anglo-Norman and continental French: the replacement of non-assertive indefinite nul by aucun. Diachronica, 28/4, 441-467.

Junker, M.-O. (1990). L'effet V1 : le verbe initial en moyen français. Revue Canadienne de Linguistique, 35/4, 351-371.

de Kok, A. (1985). La place du pronom personnel régime conjoint en français. Une étude diachronique. Rodopi : Amsterdam.

Kibbee, D. (1996). Emigrant languages and acculturation: the case of Anglo-French. The origins and development of emigrant languages ed. H. Nielsen \& L. Schøsler, 1-20. Odense: Danemark.

Leach, A. (1915). Schools of medieval England. Londres: Methuen.

Legge, D. (1950).Anglo-Norman in the Cloisters. Édimbourg: University Press.

Lusignan, S. (1986). Parler vulgairement. Les intellectuels et la langue française aux XIIIe et XIVe siècles.

Paris : Librairie académique Vrin.

Legendre, G. (1989). Inversion with Certain French Experiencer Verbs, Language 65/4, 752-782.

Lemieux, M. (1991). Le role de et dans les constructions à sujet nul et à sujet postposé en moyen français, VII ${ }^{\text {ème }}$ Colloque International sur le Moyen Français, Université de Gand.

Marchello-Nizia, Chr. (1979/1997). Histoire de la langue Française au XIV et au XV Siecles. Paris : Nathan.

Meyer-Lübke, W. (1900). Grammaire des langues romanes, tome IV: syntaxe. Paris : Welter.

Orme, N. (1973). English Schools in the Middle Ages. Londres: Routledge.

Prévost, S. (2001). La postposition du sujet en Français aux XVème et XVIème siècles, Paris: CNRS éditions.

Rothwell, W. (1976). The role of French in thirteenth-century England. Bulletin of the John Rylands University Library of Manchester 58, 445-466.

Russo, M. (2000). Origine protoromanze dell'intransitivizzazione e dell'inversione soggetto-verbo. Zeitschrift für romanische Philologie, 116, 369-417.

Skårup, P. (1975). Les premières zones de la proposition en ancien Français. Essai de syntaxe de Position. Revue Romane, numéro spécial 6, Ėtudes Romanes de l'Université de Copenhague.

Trotter, D. (2003). Not as eccentric as it looks: Anglo-French and French French. Forum for 
Modern Language Studies 39, 427-438.

Vance, B. (1993). Verb-first declaratives introduced by et and the position of pro in Old and Middle French. Lingua 89, 281-314.

Vance, B. (1997). Syntactic change in Medieval French. Dordrecht: Kluwer.

Vising, J. (1923). Anglo Norman Language and Literature. Oxford: Oxford University Press.

${ }_{1}^{1}$ À l'exception des zones celtophones.

${ }^{2}$ Supposer, comme l'a fait Rothwell, qu'à l'époque en question, vers 1300, le français ait été enseigné en tant que matière scolaire est une hypothèse qui ne repose en définitive sur aucun fondement empirique. Ce n'est que vers la fin du XIVème siècle que l'on observe la production de manuscrits compilés en vue de l'apprentissage du français (Lusignan 1986).

${ }^{3}$ Signalons au passage également la discussion de et VS chez Skårup (1975).

${ }^{4}$ Signalons pourtant le corpus CORPTEF (Guillot 2010) qui offre une vingtaine de textes en prose du XIIême siècle. 